\title{
TUNNELLED PERIPHERALLY INSERTED CENTRAL CATHERER- HOW WE DO THEM
}

\author{
Vijaynanthan $A^{1}$, Nawawi $O^{1}$, Abdullah $B^{J} J^{1}$ \\ ${ }^{1}$ Department of Biomedical Imaging, Faculty of Medicine, University of Malaya, 50603 Kuala Lumpur, Malaysia.
}

\section{Correspondence:}

Prof. Dr. Basri Johan Jeet Abdullah

Department of Biomedical Imaging, Faculty of Medicine, University of Malaya, 50603 Kuala Lumpur, Malaysia. Email: basrij@ummc.edu.my

Tel: +603 79493695

\begin{abstract}
In the current study, we report a new technique to place a tunnelled peripherally inserted central catheter (PICC) at the upper arm of patient under real-time ultrasound-guided venipuncture using disposal equipment provided within a standard PICC set. The tunnelling of the PICC required an extra time of 5 minutes but was well tolerated by all patients involved in the study. The tunnelled PICC was applied on 50 patients and the infection rate as well its catheter dwell time were compared to another 50 patients with conventional PICC. The rate of patients who developed infection decreased from $34 \%$ for conventional PICC to $16 \%$ in tunnelled PICC patients. The central line-associated blood stream infections rate was also decreased from 4.4 per 1000 catheter-days for conventional PICC to 1.3 per 1000 catheter-days for tunnelled PICC. The mean time to infection development for tunnelled PICC (24 days) was longer than those observed with conventional PICC (19 days). Tunnelled PICC has also increased the mean catheter dwell time from 27 days (for conventional PICC) to 47 days. Tunnelling a PICC has the potential to reduce the infection rate while increase the catheter dwell time.
\end{abstract}

Keywords: Tunnelled Peripherally Inserted Central Catheter, Venous Access, Central Line- Associated Blood Stream Infections, Catheter Dwell Time, Infection Rate

\section{Introduction}

With the advancement in new chemotherapeutic regimes, a better and easier venous access is becoming critical for intravenous chemotherapeutics delivery. To this end, peripheral implantable port for the arm with simpler placement, fewer complications and, in some series, greater patient acceptance has been developed as an alternative to the centrally placed chest port (1). Peripherally inserted central catheter (PICC) is widely used as alternative to the conventional central venous catheter (CVC) for providing intermediate to long term venous access, especially for long term administration of antibiotics, parenteral nutrition and chemotherapy (2). However, PICC has been related to several complications such as phlebitis, thrombosis, premature dislodgement, malfunction and central line-associated blood stream infection (CLABSI), which could have increases the cost in patient management $(3,4)$.

Tunnelling of the central catheter has been proven to improve the stability and lower the infection rate. The tunnelling of the catheter in the subcutaneous tissue during the catheter placement has decreased the catheter colonization in adults (5). Furthermore, the tunnelling of the catheter would have moved the skin exit site away from the vein entry site, which potentially reduces the extra luminal infection risk. In view of the above, tunnelling of the PICC might have improved the complications related to the conventional PICC.

We describe our technique for a tunnelled PICC using the standard PICC set and determine if catheter dwell time and infection rate of tunnelled PICC are significantly improved to conventional (non-tunnelled) PICC placements

\section{Methods}

\section{Imaging Procedure}

Using a $5-11 \mathrm{MHz}$ linear ultrasound probe, the vein to access is determined following placement of a tourniquet.

\section{Real-Time Ultrasound-Guided Venipuncture}

Figure $1 \mathrm{~A}$ shows the standard PICC set manufactured by Bard (Bard Access Systems, Salt Lake City, UT, USA), containing a single or double lumen 18-gauge silicon catheter (4F). The upper arm of patient is cleaned with antiseptic and draped. The 5-11 MHz linear ultrasound transducer (covered with sterile endoscope cover) is placed transversely over the vein and the $21 \mathrm{G}$ micro puncture needle is slowly advanced into the anterior vein wall under direct sonographic guidance. The anterior and posterior walls frequently oppose as pressure is applied and it is not 
uncommon to traverse both anterior and posterior walls simultaneously. More importantly, it is necessary to change the direction of the tip when the pressure of the needle tip displaces the vein to gain venous access.

A 0.018 " guidewire is inserted into the lumen once good flow is obtained (Figure 1B). The wire should enter the vein without hindrance or kinking. Fluoroscopy can be used to view the wire and if there is buckling, the wire should not be inserted further. Once the wire is certain to lie freely within the lumen, the wire can be further advanced. Then, the puncture site is infiltrated with local anaesthetic and the micro puncture needle is removed. A small nick is made at the access site along the wire track making sure that the skin is free. The dilator is withdrawn from the "peel away" sheath and then threated over the wire to dilate catheter tract. The proposed track for tunnelling is between 5-7 $\mathrm{cm}$ from original venous puncture site and the venous puncture site is then infiltrated with local anaesthetic using same puncture needle or a $21 \mathrm{G}$ spinal needle (Figure $1 \mathrm{C}$ ). The puncture needle is then bent gently with a more acute curve (opposite the direction of the bevel) at the distal of 2 to $3 \mathrm{~cm}$ to allow an easier exit of the needle tip at the venous entry site (Figure 1D).

Another nick is made at the distal tunnelled track near the needle entry point and the needle is directed to exit at the original venous puncture site (Figure $2 \mathrm{~A}$ ). If the access site is made capacious using blunt dissection, the tunnelling can be performed with greater ease. This can be performed at the initial stage after removal of the micro puncture needle or after track infiltration. Another technique to improve exit of the micro puncture needle after tracking is to use the blunt end of the scalpel to press the skin down. It is important to ensure that the needle path is within the subcutaneous tissue otherwise the tunnelling of the 'peel away' sheath would be difficult.

The wire is then threaded into the needle (Figure 2B) and once the tip of wire has exited the hub, the needle and wire slowly withdrawn until the wire is straight. It is important to ensure that the proximal part of the wire within the venous system does not recoil out while the entire guidewire is being straightened (Figs. $2 C$ and D). The 'peel away' sheath with the dilator is then re-threated over the wire and pressure is applied over the venous entry point to ensure there is no buckling (Figure $3 \mathrm{~A}$ ). The dilator is then withdrawn while the 'peel away' sheath is left in place. After the removal of the dilator component of the 'peel away' sheath, the PICC is introduced through the sheath and advanced into position (Figure 3B). Then, the 'peel away' sheath hub is split (Figure $3 \mathrm{C}$ ) and slowly withdrawn while ensuring the PICC does not get pulled
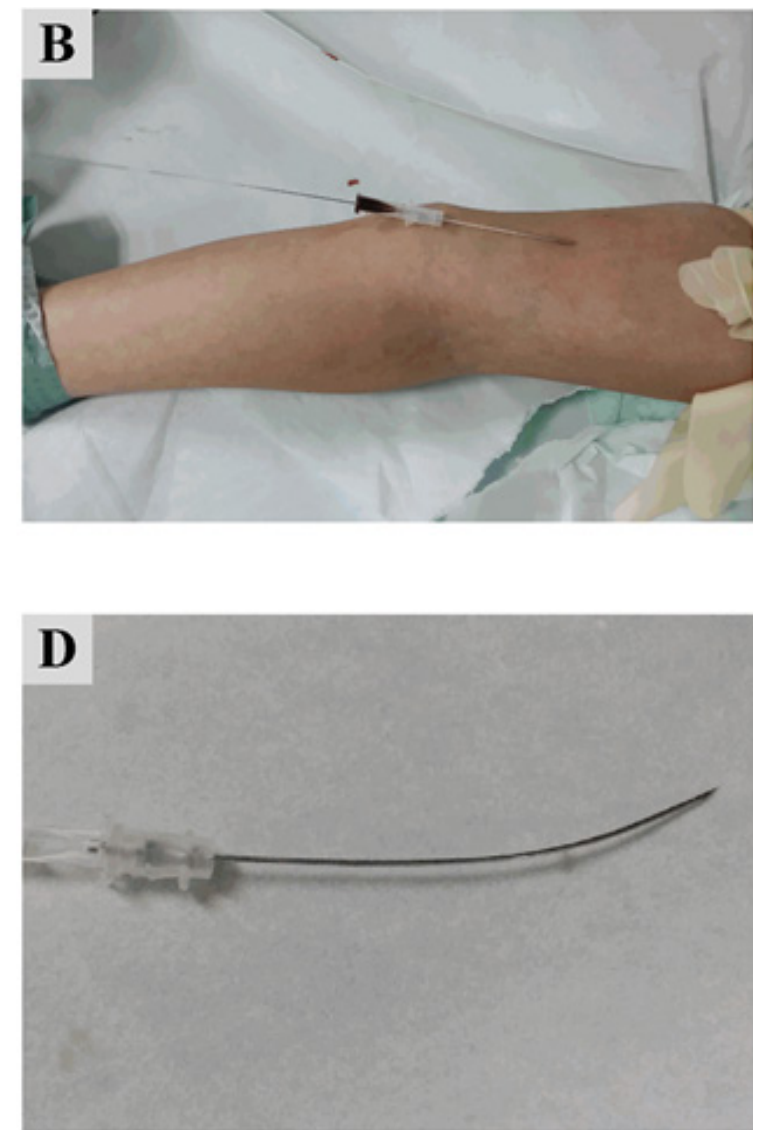

Figure 1. (A) The standard Bard PICC set and the linear ultrasound probe covered with sterile cover. (B) Puncture needle with a guidewire threaded into the vein. (C) The track being infiltrated with local anaesthetic using the same puncture needle or a $21 \mathrm{G}$ spinal needle. (D) The micro puncture needle with the tip deflected from the bevel. 

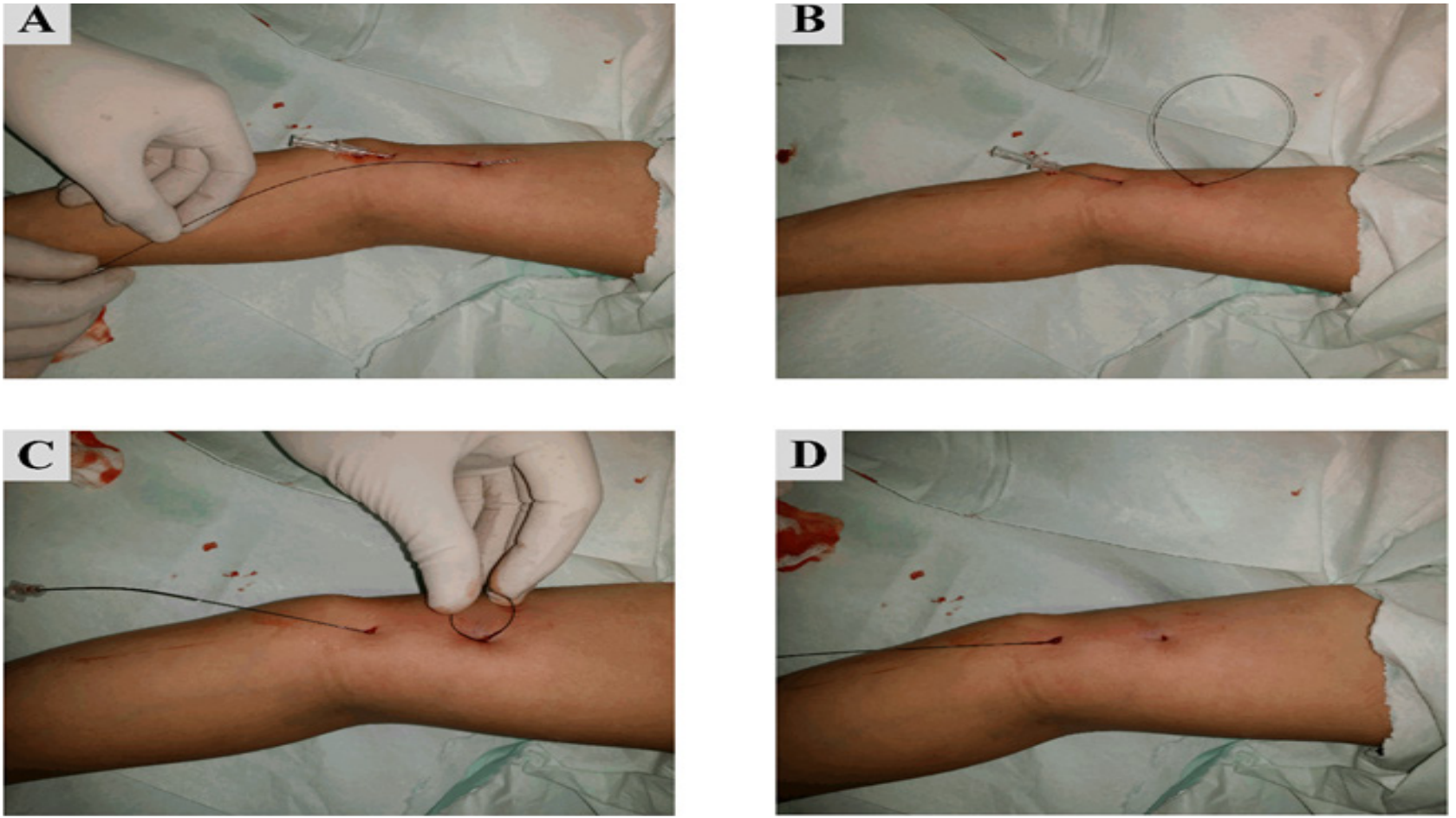

Figure 2. (A) Needle tip exiting the original puncture site. (B) The guidewire threaded into the needle as it is slowly withdrawn. (C) The operator has to ensure that the proximal portion of the wire does not recoil out from the vein while the entire guidewire is being straightened. (D) The wire after it has been straightened with the portion tunnelled in the subcutaneous tissue.
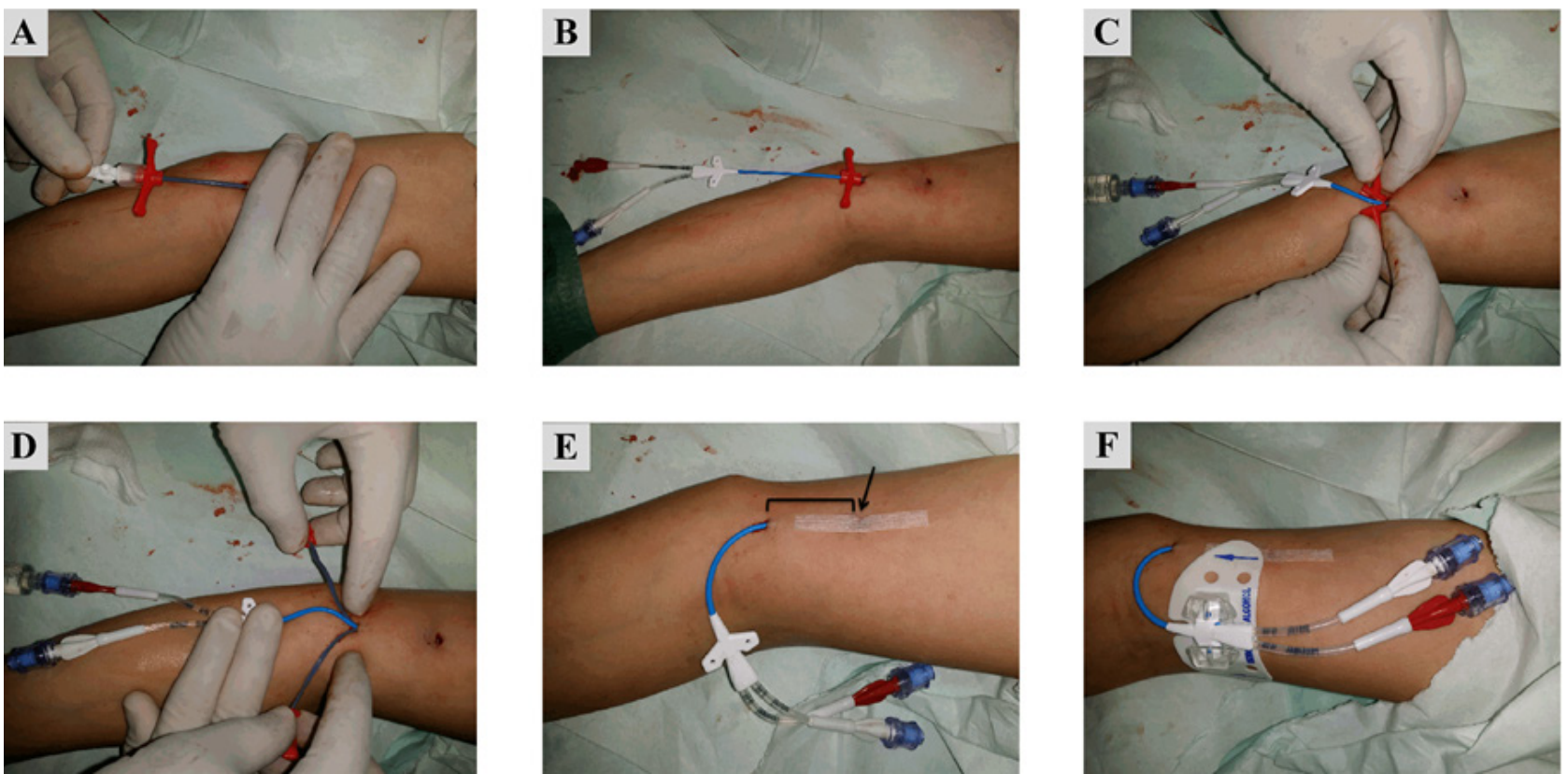

Figure 3. (A) Pressure is being applied at the venous access site to reduce buckling of the peel away sheath as it traverses the initial venous access. (B) Double lumen Groschong catheter inserted through the peel away sheath. (C) The peel away sheath hub is split. (D) The peel away sheath is slowly withdrawn while ensuring the PICC does not get pulled back by holding it down by an assistant. (E) The initial venous access site (black arrow) is closed using Steri-strips after ensuring that there is no oozing from the site and the bracket shows the length of the tunnelling. The entire set-up is then covered with OPSITE film. (F) The PICC anchored using the Stadlock and another Steri-strip can be placed at the distal end of the subcutaneous tunnel. 
back by holding it down by an assistant (Figure 3D). The position of the PICC can be checked using fluoroscopy, if necessary. The initial venous access site, as shown by a black arrow in Figure 3E, is closed using a Steri-Strip after ensuring there is no oozing from the site. The PICC is anchored using the Stadlock and another Steri-Strip can be placed at the distal end of the subcutaneous tunnel as shown in Figure 3F.

\section{Study Protocol}

We prospectively performed conventional PICC and tunnelled PICC on two separate patient groups of 50 patients each. The type of PICCs used in this study is given in Table 1. Real-Time Ultrasound-Guided Venipuncture is a standard of care for venous access. All patients were reviewed until either the occurrence of PICC-related complication necessitated removal, completion of therapy, death or till the end of the study. The CLABSI was confirmed in each case by demonstrating concordance between isolates colonizing the PICC at the time of infection and from blood cultures. Written informed consent was obtained from all patients before the PICC placement.

Table 1. Type of PICC used in the study for both control and tunnelled PICC patient groups.

\begin{tabular}{lcc}
\hline Types of PICC & Control [No. (\%)] & $\begin{array}{c}\text { Tunneled [No } \\
(\%)]\end{array}$ \\
\hline $\begin{array}{l}\text { Double lumen } \\
\text { Groshong }\end{array}$ & $32(64 \%)$ & $30(60 \%)$ \\
Single lumen Groshong & $9(18 \%)$ & $12(24 \%)$ \\
Single lumen Polyrad & $9(18 \%)$ & $8(16 \%)$ \\
\hline
\end{tabular}

The commonest indication of PICC in both groups was for antibiotic administration (60\% in non tunneled group and $50 \%$ in tunneled group), followed by chemotherapy ( $25 \%$ in non-tunneled group and $30 \%$ in tunneled), total parenteral nutrition and IV access. Patients who were diagnosed with infection made up the majority of the study population for both groups (58\% in non-tunneled and $50 \%$ in tunneled), followed by malignancy ( $36 \%$ in non-tunneled and $46 \%$ in tunneled) and others (scleroderma, mycotic aneurysm and Chron's disease).

\section{Results}

The mean catheter dwell time for tunnelled PICC at 47 days ( 2352 catheter-days for 50 tunnelled PICC) was significantly longer than conventional PICC at 27 days (1355 catheter days for 50 conventional PICC). Tunnelling of the PICC has decreased the infection rate from $34 \%$ for conventional PICC to $16 \%$ for tunnelled PICC. The CLABSI rate for patient with tunnelled PICC at $6 \%$ was also lower than the CLABSI rate for conventional PICC at $12 \%$. The mean time to infection development for tunnelled PICC at 24 days was found longer than the conventional PICC (19 days). However there was no significant difference in the mean time to development of infection between the 2 groups $(p=0.7)$. The catheter removal due to infection was $24 \%$ in conventional PICC patients but was significantly reduced to $4 \%$ in tunnelled PICC ( $p=0.002)$.

All CLABSI cases occurred in patients with double lumen PICC. Patients with hematological malignancy requiring PICC for chemotherapy were more prone to develop CLABSI, forming all of the CLABSI cases in the tunneled group and $66.6 \%$ of the CLABSI cases in the non-tunneled group (odds ratio 7.6, relative risk of 6). No significant difference was however seen between the 2 groups. Patients with previous PICC were more likely to develop CLABSI compared to patients with no previous PICC [ 5 cases (19.2\%) compared to 4 cases $(5.4 \%)$ respectively, $p$ value $=0.049$, relative risk of 3.56 , odds ratio of 4.16$)]$.

\section{Discussion}

The mean catheter dwell time for tunnelled PICC was found significantly higher than those observed with conventional PICC. Our results seems to be contradicted with previous reports suggesting longer catheter dwell time would have posed a higher risk of infection $(6,7)$. While all infections were diagnosed during long term follow-up, there were no procedure-related early infections (within 1 week after the procedure) observed with tunnelled PICC patients. In fact, the tunnelled PICC has a longer mean time to infection development compared to conventional PICC. There was no major procedural complications from the technique as no incidence of vascular injury or excessive bleeding, mechanical complications (such as catheter occlusion, fracture or malposition), 'pinch-off' or compression point in the catheter line at the venous entry in tunnelled PICC patients. Furthermore, other major complications such as infection or venous thrombosis were found equivalent in both conventional PICC and tunnelled PICC patients.

Overall, the technical success of inserting a tunnelled PICC in patient is high despite required an extra average time of 5 minutes to create the tunnel. However, the tunnelling procedure was very well tolerated by patients and the skin of the arm is easily withstands the tunnelling process. Additionally, the procedure to create a tunnel in the arm of the patient is much simpler and required a shorter procedural time than in the chest. However, the venous access for tunnelled PICC has to be made in the mid-forearm of the patient to avoid tunnelling across the joint has limited the patient's ability to use the arm freely. Furthermore, mechanical compression, kinking or mitigation of the PICC may occur during falling. Further improvement of the tunnelled PICC with the use of the modified cuffed PICC as described previously (8) could have improves its stability.

Tunnelling a PICC has the potential to reduce the infection rate while increase the catheter dwell time, provided all other measures recommended for PICC care are implemented. As the technique of tunnelling PICC becomes more widely applied, the benefits such as pain elimination of routine peripheral intravenous access and 
lower risk of venous inflammation, venous thrombosis and extravasation of cytotoxic agents during chemotherapy will be available to more patients. This will reduce the need of repeated PICC insertions due to infection. As more and more care is shifted towards outpatient care (continuous infusion chemotherapy) and in-home care (palliative medicine), the tunnelled PICC not only reduces the medical cost but also meets the increased societal demand for outpatient cancer chemotherapy. The tunnelled PICC could potentially reduce the need to perform routine central venous port implantation procedures.

\section{Conclusions}

We have described our simple technique of tunnelling PICC using the Bard system without the needs of any additional expensive or disposal equipment. We believe any operator with moderate expertise can perform this technique following the procedure described. Further improvements in the PICC with the addition of cuffs would enable the tunnelled PICC to be used for multiple functions, which can contribute to a secure, safe and seamless care from anti-cancer therapy to palliative medicine. We hope this procedure will become more common and eventually be validated in prospective multicentre randomized clinical trials regarding its non-inferiority or superiority to other central venous line procedures e.g. PICC with respect to safety.

\section{Conflict of Interests}

The authors declare no conflict of interest.

\section{References}

1. Foley MJ. Radiologic Placement of Long-term Central Venous Peripheral Access System Ports (PAS Port): Results in 150 Patients. J Vasc Interv Radiol. 1995; 6(2):255-62.

2. Merrell SW, Peatross BG, Grossman MD, Sullivan JJ, Harker WG. Peripherally inserted central venous catheters. Low-risk alternatives for ongoing venous access. West J Med. 1994; 160(1):25-30.

3. Mermel LA. Prevention of intravascular catheterrelated infections. Ann Intern Med. 2000; 132(5):391402.

4. Warren DK, Quadir WW, Hollenbeak CS, Elward AM, Cox MJ, Fraser VJ. Attributable cost of catheterassociated bloodstream infections among intensive care patients in a nonteaching hospital. Crit Care Med. 2006; 34(8):2084-9.

5. Nahum E, Levy I, Katz J, Samra Z, Ashkenazi S, BenAri J, et al. Efficacy of subcutaneous tunneling for prevention of bacterial colonization of femoral central venous catheters in critically ill children. Pediatr Infect Dis J. 2002; 21(11):1000-4.

6. Balkhy HH, Alsaif S, El-Saed A, Khawajah M, Dichinee $\mathrm{R}$, Memish ZA. Neonatal rates and risk factors of device-associated bloodstream infection in a tertiary care center in Saudi Arabia. Am J Infect Control. 2010; 38(2):159-61.
7. McLaws ML, Berry G. Nonuniform risk of bloodstream infection with increasing central venous catheter-days. Infect Control Hosp Epidemiol. 2005; 26(8):715-9.

8. Selby Jr, Cohn DJ, B. GKJ. Peripherally inserted tunnelled catheters: a new option for venous access. Minim Invasive Ther Allied Technol. 2001; 10(45):231-4. 